\title{
Enhanced Concentration of Polarizable Anions at the Liquid Water Surface: SHG Spectroscopy and MD Simulations of Sodium Thiocyanide
}

\author{
Poul B. Petersen and Richard J. Saykally* \\ Department of Chemistry, University of California, Berkeley, California 94720
}

\author{
Martin Mucha and Pavel Jungwirth* \\ Institute of Organic Chemistry and Biochemistry, Academy of Sciences of the Czech Republic and Center for \\ Biomolecules and Complex Molecular Systems, Flemingovo nam. 2, 16610 Prague 6, Czech Republic
}

Received: February 18, 2005; In Final Form: March 28, 2005

Contrasting current textbook descriptions, a consistent picture of substantial concentration enhancement of highly polarizable anions at the surface of aqueous electrolyte solutions is emerging. Such enhancement may have important implications for chemistry occurring on aqueous aerosols and ocean surfaces. Here we present a combined experimental and theoretical investigation of the liquid/air interface of aqueous sodium thiocyanide at varying salt concentrations. Normalized second harmonic generation intensities fitted to Langmuir isotherms yield a Gibbs free energy of adsorption of $-1.80 \mathrm{kcal} / \mathrm{mol}$. These results are in accord with molecular dynamics simulations in slab geometry, which predict an appreciable surface enhancement of $\mathrm{SCN}^{-}$.

\section{Introduction}

Recent theoretical and experimental studies have indicated the adsorption of highly polarizable anions to the outermost layer of the liquid water-air interface. ${ }^{1-9}$ These findings contradict the textbook description of ions being repelled from the interface and the outermost surface layer being devoid of ions. ${ }^{10-12}$ This conventional wisdom is largely based on macroscopic measurements of the increasing surface tension of aqueous solutions with salt concentration. When combined with the Gibbs adsorption equation, such measurements dictate that the ion concentration, integrated over the entire interfacial region, must be depleted with respect to the bulk. This conclusion has been rationalized by electrostatic continuum models dating back to Onsager and Samaras, wherein the interface is described as a discontinuity between two continuous dielectric media. ${ }^{13}$ Recent state-of-the-art continuum models have been augmented to include most interfacial forces. ${ }^{14-19}$ These modern models mostly depict the outermost liquid layer of the interface as depleted of ions; however, in specific cases (e.g., for highly polarizable ions) some of them predict ion adsorption at the aqueous surface. While being useful in many respects, continuum models still engender several theoretical objections, such as problems with an accurate description of interactions within the first solvation shell, as described in a recent overview. ${ }^{20}$

The picture of an essentially ion-free surface layer was challenged a few years ago by the atmospheric community, which proposed that chemical reactions on aqueous sea salt particles, ocean surfaces, and laboratory aerosol experiments could not be explained without invoking ions at the surface. ${ }^{21-23}$ This motivated studies by molecular dynamics simulations with use of polarizable potentials, ${ }^{1-5,22}$ followed by indirect experi-

* Address correspondence to these authors. R.J.S.: phone +1 (510) 642 8269, fax +1 (510) 642-8369, e-mail saykally@ berkeley.edu. P.J.: phone +420 (220) 410 314, fax +420 (220) 410 320, e-mail pavel.jungwirth@ uochb.cas.cz. mental investigations by Sum-Frequency Generation $(\mathrm{SFG})^{8,24}$ and later by direct measurements with Second Harmonic Generation $(\mathrm{SHG})^{6,7}$ of highly polarizable anions at the liquid water-air interface.

It is important to understand that the above theoretical and experimental findings do not contradict the macroscopic surface tension measurements and thermodynamic arguments. The macroscopic measurements offer no detailed molecular description of the surface concentration, but only dictate that the integrated ion concentration over the entire interfacial region is depleted. The highly polarizable anions show a very nonmonotonic interfacial distribution, strongly enhanced in the outermost liquid layer but depleted in the subsurface. Qualitatively, this is emerging from the molecular dynamics simulations, although the subsurface depletion is not quantitative due to the finite width of the "bulk" region of the simulated slab systems. ${ }^{25}$ The surface propensity of large polarizable anions can be contrasted to the behavior of small nonpolarizable ions (such as alkali cations or fluoride), which are indeed repelled from aqueous surfaces, in accord with the traditional view.

Here we present a combined experimental and theoretical investigation of the surface adsorption of the thiocyanide anion in aqueous solutions of NaSCN of varying concentrations. Our experiments exploit the surface specific nature of SHG spectroscopy and directly probe the $\mathrm{SCN}^{-}$ions via the strong chargetransfer-to-solvent (CTTS) transitions in the UV. The experimental SHG investigations were designed to measure the surface concentration dependence on the bulk concentration. The surface concentration was then fit to the Langmuir model and the Gibbs free energy of adsorption was determined. It is worth noting that the SHG experiments can only establish a minimum in the Gibbs free energy profile in the interfacial region, and thus a nonmonotonic anion distribution. The actual position of this minimum, at or just below the outermost surface layer, must rely on either anisotropy arguments or theoretical investigations. Our molecular dynamics simulations of solutions in a slab 
geometry provide this detailed molecular insight not obtainable in the experiments. The simulations show the concentration enhancement of the anions occurring in the outermost surface layer of the interface.

\section{Experimental Details and the Langmuir Model}

A detailed description of our SHG experiments is given elsewhere ${ }^{26}$ and only a brief account is given here. The femtosecond laser system consists of a home-built Ti:sapphire oscillator and a commercial regenerative amplifier (Spectra Physics, Spitfire) that pumps two OPAs (Light Conversion, TOPAS). The laser system is capable of generating sub-100 fs laser pulses in the range $290 \mathrm{~nm}$ to $10 \mu \mathrm{m}$. The output of the TOPAS is purified with dichroic mirrors and optical filters before being focused onto the sample at $45^{\circ}$. The SHG beam is separated from the fundamental by dichroic mirrors and a prism before being collected on a solar blind PMT. Due to the strong SHG response of thiocyanide, pulse energies of only $0.5-3 \mu \mathrm{J}$ are needed for the experiments.

The sample is kept in an enclosed box purged with pure nitrogen that also gently stirs the liquid surface. All glassware in contact with the sample is cleaned with Nochromix (a hot chromic acid substitute) and rinsed with $18.2 \mathrm{M} \Omega$ water before each experiment. All solutions are made with $99.99 \%$ pure $\mathrm{NaSCN}$ and ultrapure water $(18.2 \mathrm{M} \Omega$ and $\leq 4 \mathrm{ppb}$ total organic content) from a water purification system (Millipore, Milli-Q Gradient).

As a second-order optical process, SHG is forbidden in bulk centrosymmetric media within the dipole approximation, and is thus a surface specific technique for liquids. ${ }^{27,28} \mathrm{SHG}$ and the related SFG process have been well characterized as direct probes of the liquid water surface structure and surfactants thereon, ${ }^{29,30}$ with probing depth of a few molecular layers. ${ }^{31}$ The SHG intensity $\left(I_{2 \omega}\right)$ is described in terms of the secondorder susceptibility of the surface $\left(\chi^{(2)}\right)$, which, in turn, is the sum of the molecular hyperpolarizabilities $(\beta)$ in the interfacial region:

$$
\begin{gathered}
I_{2 \omega} \propto\left|\chi^{(2)}\right|^{2} \times I_{\omega}^{2} \\
\chi^{(2)}=\chi_{\text {water }}^{(2)}+\chi_{\mathrm{SCN}^{-}}^{(2)} \\
\chi_{\mathrm{SCN}^{-}}^{(2)}=\sum_{i} \beta_{i}=N_{\mathrm{S}} \times\langle\beta\rangle_{\text {Orientation }}
\end{gathered}
$$

Like linear dielectric constants, second-order susceptibilities are real at nonresonant frequencies but become complex near resonances. For the experiments presented here, the water susceptibility is nonresonant (and thus real), but the thiocyanide resonance contains a complex, wavelength-dependent phase. If the orientation of the surface species and the magnitude of the hyperpolarizability are assumed to be constant over the concentration range studied, the change in the SHG intensity can be related to the change in surface concentration. This may seem a strong assumption, given the high concentrations studied, implying significant ion-ion interactions. However, the simulations show identical surface profiles for $\mathrm{S}, \mathrm{C}$, and $\mathrm{N}$, implying that the $\mathrm{SCN}^{-}$anions lie flat at the surface at all concentrations. Furthermore, previous SHG investigations of the Langmuir behavior of surfactants have shown that corrections due to changes in the molecular orientation are relatively small $(<25 \%)$. $^{32}$

The surface concentration of the ions is represented by the Langmuir adsorption isotherm, which is a standard and well- characterized adsorption model for liquid surfaces. ${ }^{10,33-35}$

$$
N_{\mathrm{S}}=N_{\mathrm{S}}^{\max } \frac{K x}{K x+C_{\mathrm{w}}} \approx \frac{N_{\mathrm{S}}^{\mathrm{max}} \times x}{x+55.5 M \exp \left(\Delta G_{\mathrm{Ads}} / R T\right)}
$$

Here $N_{\mathrm{S}}^{\max }$ is the maximum surface concentration, $K$ is the equilibrium constant for surface adsorption, $x$ and $C_{\mathrm{w}}$ are the bulk solute and water concentration, respectively, and $\Delta G_{\mathrm{Ads}}$ is the Gibbs free energy of adsorption.

The Langmuir model assumes a maximum of one monolayer coverage $\left(N_{\mathrm{S}}^{\max }\right)$ and negligible surfactant-surfactant interactions. At low concentration, ions form a somewhat diffuse layer at the interface, but at higher concentrations, the simulations show the concentration enhancement to occur in a narrow region about $3 \AA$ wide at the outermost surface layer. The enhancement is then followed by depletion in a broader region over deeper layers, with the total interfacial region having a width of more than $10 \AA$. The probing depth of the experiment is currently not well-known. For the pure water interface with a 90-10 thickness of approximately $5 \AA$, calculations have shown that almost the entire SHG intensity comes from the outermost surface layer. ${ }^{31}$ For the salt solutions, the interface is wider (around $10 \AA$ ) and the SHG intensity is, in principle, generated over this entire range that shows a net asymmetry. However, the simulation shows that concentration enhancement in the outermost surface layer coincides with the 90-10 region of the water density profile. This region thus experiences a much higher asymmetry and will dominate the SHG response. The inferred Gibbs free energy should be viewed as a weighted average over the ions within the probing depth of the experiment.

The Langmuir model also assumes negligible surfactantsurfactant interactions or at least similar surfactant-surfactant and surfactant-solvent interactions. For the ions studied here, this assumption is likely not to be fully justified. However, the Debye length is $6 \AA$ for a $0.25 \mathrm{M}$ solution of a $1: 1$ electrolyte, therefore, long-range electrostatic interactions between ions are well screened at the high concentrations studied here and close range interactions will dominate. Details concerning the close range interactions, such as dispersion and polarization, are not very well understood for electrolyte solutions and the differences between ion-ion and ion-water interactions could prove important at high concentrations. ${ }^{20}$ This would result in a concentration-dependent Gibbs free energy of adsorption. Despite these potential obstacles, the Langmuir model is observed to fit the data very well and is, therefore, used due to its simplicity, rather than adopting a more complicated model.

Denoting the real water susceptibility as $A$ and the real and imaginary parts of the thiocyanide susceptibility as $B$ and $C$, respectively, we incorporate the Langmuir model for the surface concentration into the expression for the SHG intensity:

$$
\begin{aligned}
I_{\mathrm{SHG}} & \propto\left|A+(B+i C) \times N_{\mathrm{S}}\right|^{2} \\
& =\left(A+B \times N_{\mathrm{S}}\right)^{2}+\left(C \times N_{\mathrm{S}}\right)^{2} \\
& =\left(A+\frac{\left(B \times N_{\mathrm{S}}^{\max }\right) \times x}{x+D}\right)^{2}+\left(\frac{\left(C \times N_{\mathrm{S}}^{\max }\right) \times x}{x+D}\right)^{2}
\end{aligned}
$$

Here, $D$ is the Langmuir constant $55.5 M \exp \left(\Delta G_{\text {Ads }} / R T\right)$. The SHG intensities at different wavelengths are fit simultaneously to this equation, allowing the real and imaginary parts of the thiocyanide susceptibility ( $B$ and $C$ ) to change with the wavelength, while keeping $D$ fixed for all wavelengths. 
TABLE 1: Partial Charges, $q$, Lennard-Jones Radii, $R$, Lennard-Jones Well Depths, $\epsilon$, and Atomic Polarizabilities, $\alpha$, of the Atomic Sites for $\mathrm{Na}^{+}$and $\mathrm{SCN}^{-}$

\begin{tabular}{ccccc}
\hline atom & $q[\mathrm{au}]$ & $R[\AA]$ & $\epsilon[\mathrm{kcal} / \mathrm{mol}]$ & $\alpha\left[\AA^{3}\right]$ \\
\hline $\mathrm{Na}$ & +1 & 1.3190 & 0.1300 & 0.24 \\
$\mathrm{~S}$ & -0.75 & 2.1500 & 0.3639 & 2.0 \\
$\mathrm{C}$ & 0.49 & 1.8801 & 0.1016 & 2.0 \\
$\mathrm{~N}$ & -0.74 & 2.0750 & 0.0741 & 2.0
\end{tabular}

\section{Computational Details}

The simulated systems consisted of 864 water molecules and 9,18 , or $54 \mathrm{NaSCN}$ ion pairs corresponding to $0.6,1.2$, and 3.5 M solutions, respectively. The ions and molecules were placed in a simulation box with dimensions $30 \times 30 \times 150 \AA^{3}$ and periodic boundary conditions were employed. Due to the elongated $z$-length of the box an infinite liquid slab was formed in the middle of the $z$-dimension with two planar liquid-vapor interfaces. ${ }^{36}$

The water model used is the polarizable POL3 water. ${ }^{37}$ The Lennard-Jones parameters, partial charges, and atomic polarizabilities for sodium and thiocyanate ions are given in Table 1.

The parameters for the sodium anion are taken from ref 38 . The partial charges of thiocyanate were determined by the CHELPG procedure ${ }^{39}$ from the wave function calculated the MP2/aug-cc-pvdz level. The Lennard-Jones parameters were chosen such as to reproduce the ab initio geometry and intermolecular binding energy of the $\mathrm{SCN}^{-} \cdot \cdots \mathrm{H}_{2} \mathrm{O}$ cluster. ${ }^{40}$ Nonbonded interactions were cut off at $12 \AA$ and long-range electrostatics was treated by the Particle Mesh Ewald technique, ${ }^{41}$ with a grid size of $1 \AA$.

The polarizability was first evaluated in the gas phase at the MP2/aug-cc-pvdz level. Diagonal elements of the polarizability tensor are $\alpha_{x x}=\alpha_{y y}=5.79 \AA^{3}$, and $\alpha_{z z}=11.6 \AA^{3}$. However, for the sake of the force field implementation, a single value of the isotropic polarizability should be given, which is the average of the diagonal values, $\alpha=7.7 \AA^{3}$. Moreover, this value should be scaled down by about $10-25 \%$ to account for solvent effects $^{4,42}$ and distributed among the atoms (see Table 1). Also, to avoid the well-known polarization catastrophe during the MD simulation, ${ }^{43}$ we adopted a scheme that effectively scales down the atomic polarizability once the instantaneous electric field at the point overcomes a certain limit. To be more specific, the functional dependence, $p=p(E)$, of the induced dipole with respect to the applied electric field strength is no longer linear, $p=\alpha E$, but has a more complex form (see Figure 1). We can divide the induced dipole dependence into three regions: (1) a linear regime for small fields, $E<E_{1}$, (2) a constant regime for strong fields, $E>E_{2}$, where the induced dipole does not further rise with increasing electric field, but remains constant at the value $p_{\max }$, and (3) a region of intermediate fields, $E_{1}<$ $E<E_{2}$, where the dependence is quadratic, such that the whole curve is smooth. Then, only two parameters have to be given, to specify the functional form completely, i.e., the maximum induced dipole, $\alpha E_{\max }$, and the induced dipole at the end of linear regime, $\alpha E_{1}$. We have used values of $E_{\max }=0.211 \mathrm{D}^{-3}$, and $E_{1}=0.19 \mathrm{D}^{-3}$. The values were chosen such that the scaling does not affect the behavior of pure POL3 water.

After a period of equilibration of at least $500 \mathrm{ps,} \mathrm{production}$ simulations were run for 2 ns with a time step of $2 \mathrm{fs}$. Temperature was kept constant at $300 \mathrm{~K}$ by means of the Berendsen thermostat. ${ }^{44}$ All bond lengths were constrained by the SHAKE algorithm. ${ }^{45}$ The atomic positions were recorded every 2 ps, and histogramed according to their $z$-coordinate and atomic type, giving the time-averaged density profiles. The MD

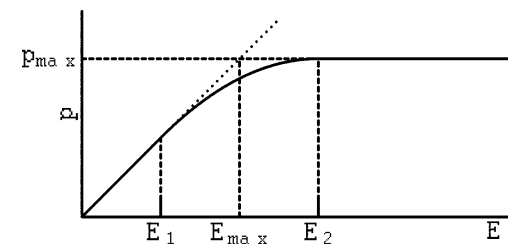

Figure 1. Scaling down of the polarizability, with increasing electric field strength. The induced dipole, $p$, depends on the electric field strength, $E$ : linear for $E<E_{1}$, quadratic for $E_{1}<E<E_{2}$, and constant for $E>E_{2}$. The induced dipole has an upper bound, $p_{\max }$.

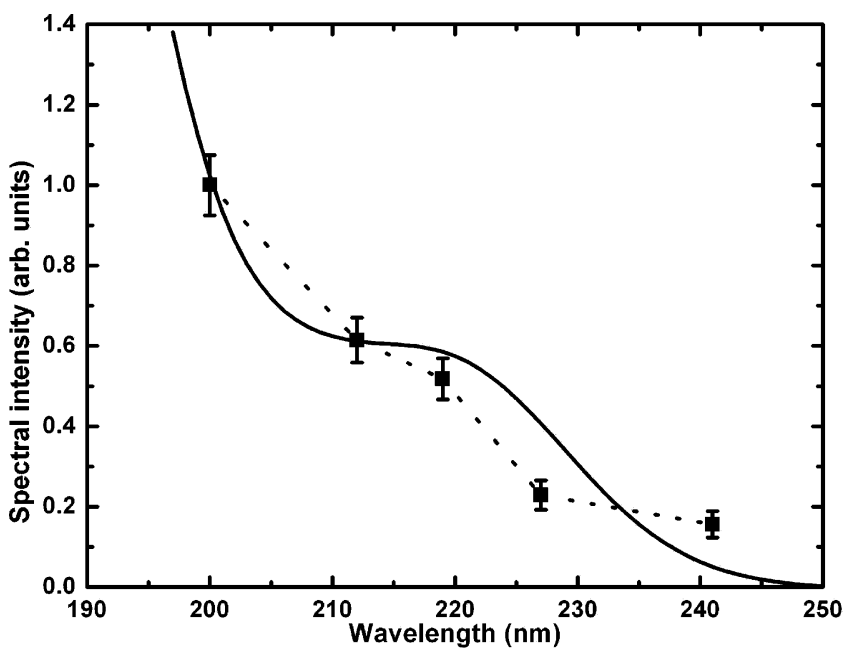

Figure 2. Spectral dependence of the $\mathrm{SCN}^{-} \mathrm{CTTS}$ spectrum. The solid line is the bulk absorbance spectrum, showing two CTTS transitions. The solid squares are the magnitudes of the second-order response of thiocyanide $\left(\left|\chi_{\mathrm{SCN}}^{(2)}\right|\right)$ and are thus representative of the surface spectrum. A small blue shift $(5-10 \mathrm{~nm})$ of the surface spectrum is observed with respect to the bulk due to the interactions occurring in the dense ionic double layer.

simulations were performed with the AMBER 8 program package $^{46}$ and the ab initio calculations were done with the Gaussian 03 suite of programs. ${ }^{47}$

\section{Experimental Results}

The thiocyanide anion exhibits strong $\left(\epsilon \sim 10^{4} \mathrm{M}^{-1} \mathrm{~cm}^{-1}\right)$ charge-transfer-to-solvent (CTTS) transitions in the UV, as shown in Figure 2. CTTS transitions also exhibit a large nonlinear response that can be used to directly probe the surface concentration of the anion as described in Section 2. The concentration dependence of the SHG intensity at different wavelengths is shown in Figure 3. A small initial decrease in the SHG intensity is observed at 227 and $241 \mathrm{~nm}$ due to destructive interference of the thiocyanide susceptibility with the water background. This initial decrease is consistent with the high concentration increase and is not indicative of the Jones-Ray effect ${ }^{48,49}$ that we observe for iodide 6 and ferrocyanide. ${ }^{50}$ That the Jones-Ray effect is not observed for thiocyanide or azide ${ }^{7}$ is itself very interesting and could be due to the conjugated bonds of these ions that are not present in the simple ions such as iodide or complex ions such as ferrocyanide. Jones and Ray observed a surface tension minimum for potassium thiocyanide, but the minimum was smaller and occurred at higher concentrations than for the other salts. The small surface tension decrease could be due to an artifact as argued by Langmuir ${ }^{51,52}$ or due to some other aspects of the poorly understood Jones-Ray effect. However, a small initial offset in the SHG intensity of these anions of 0.05 due to the Jones-Ray effect cannot be excluded. 
a)

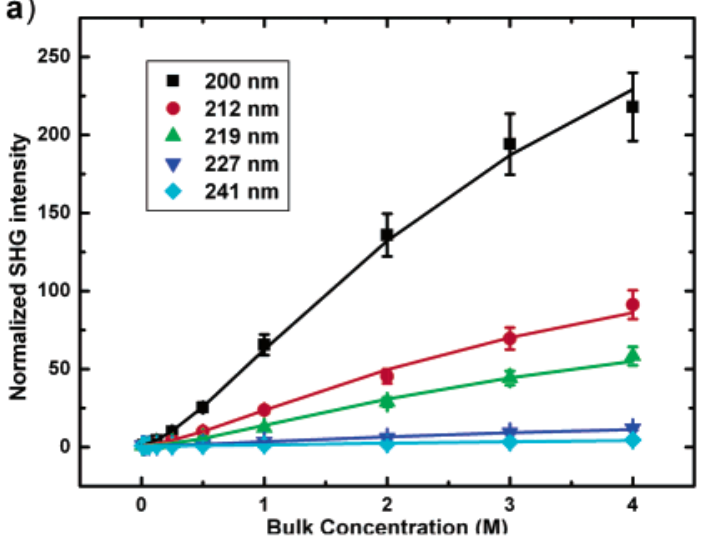

b)

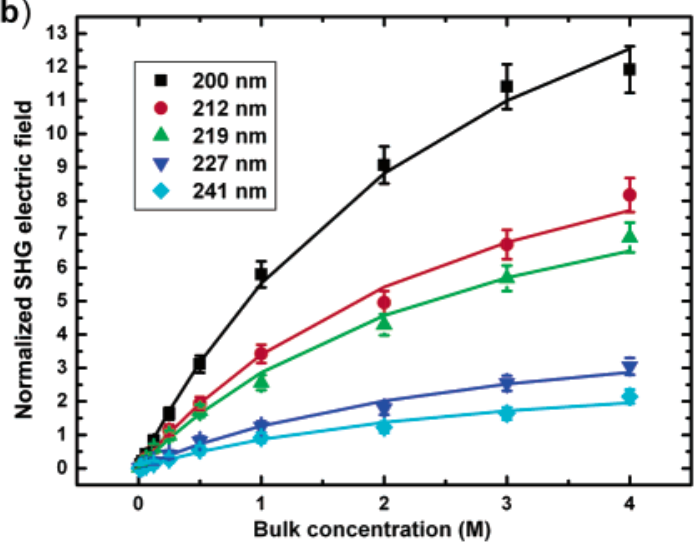

Figure 3. SHG response at five different wavelengths. Panel a shows the SHG intensity of NaSCN solutions normalized to that of pure water. Each wavelength is fit to the Langmuir model, yielding a common Gibbs free energy of adsorption of $-1.80 \pm 0.03 \mathrm{kcal} / \mathrm{mol}$. The different intensities reflect the spectral dependence, shown in Figure 2. Panel b shows the extracted SHG electric field generated by thiocyanide normalized to the response of pure water. The surface concentration of thiocyanide is proportional to the SHG electric field, and given the (currently unknown) relative second-order cross-section between water and thiocyanide and the water surface concentration, could be calculated in ions/area. The SHG electric field is thus representative of a standard Langmuir plot with the magnitude at each wavelength reflecting the surface spectrum.

The SHG intensity is fit to an expression incorporating the Langmuir model, as described by eq 5. Each wavelength is fit to an individual complex thiocyanide susceptibility, but all are fit with a common Langmuir constant. The magnitude of the thiocyanide susceptibility $\left(\sqrt{B^{2}+C^{2}}\right)$ for the different wavelengths, is shown, along with the bulk absorption spectrum in Figure 2. A blue shift of this high concentration surface spectrum with respect to the bulk is observed. A similar blue shift at high concentrations has been observed for sodium and potassium iodide $^{6}$ as well as for hydroiodic acid, ${ }^{53}$ but not for azide, ${ }^{7}$ and is in contrast to the red shift observed at dilute concentrations due to the less polar nature of the water surface. ${ }^{54}$ The high concentration blue shift is thus indicative of significant ionion interactions occurring at the interface.

The surface concentration of thiocyanide is proportional to the SHG electric field generated by thiocyanide, which can be extracted from the fit. Panel $b$ in Figure 3 shows the extracted SHG electric field normalized to pure water. The curves thus represent the surface concentration with the magnitude at each wavelength reflecting the second-order cross-section, as seen in Figure 2. From the fitted Langmuir constant, a Gibbs free energy of adsorption for thiocyanide of $-1.80 \pm 0.03 \mathrm{kcal} / \mathrm{mol}$ is extracted. This is less than the energy extracted for azide

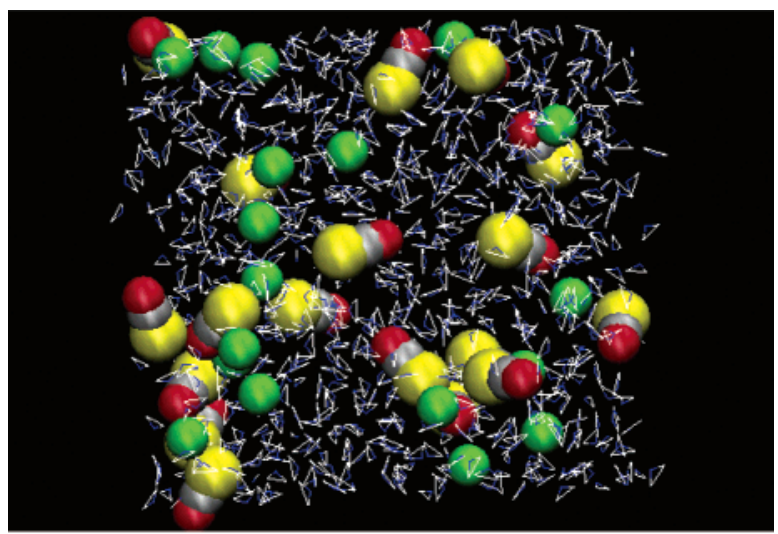

Figure 4. Typical snapshot from the MD simulation. The picture shows a side view of the simulation box, (with solution/air interfaces on the right and on the left) with water molecules depicted as triangles and the atoms of the solvated ions as balls.

$(-2.4 \pm 0.1 \mathrm{kcal} / \mathrm{mol})^{7}$ but more than that for the high concentration iodide adsorption $(<1 \mathrm{kcal} / \mathrm{mol}) .{ }^{26}$ The Gibbs free energy of $-1.80 \mathrm{kcal} / \mathrm{mol}$ for $\mathrm{SCN}^{-}$is not sufficient to ensure complete saturation of the surface before the solubility limit of the bulk is reached. However, beginning saturation, as manifested by a sublinear increase of the surface concentration with respect to the bulk, of the outermost surface layer is observed above $1 \mathrm{M}$ bulk concentration.

\section{Computational Results}

A typical snapshot of the MD simulation box (which is periodically repeated in all three dimensions) showing the aqueous slab with dissolved $\mathrm{Na}^{+}$and $\mathrm{SCN}^{-}$ions is depicted in Figure 4 . We see qualitatively that in the aqueous bulk the cations and anions are roughly evenly distributed, while at the air/water interface there is an increased concentration of thiocyanide. The figure thus demonstrates the ion-specific behavior at the air/water interface, with large polarizable ions exhibiting a propensity for the surface.

This result is quantified in panels $\mathrm{a}-\mathrm{c}$ of Figure 5 which show the density profiles across the slab of the $0.6,1.2$, and 3.5 M solution, respectively. All these density profiles represent statistically averaged values over the 2 ns simulations. Each curve corresponds to an individual species, normalized so that the area under all the curves is the same and the bulk water density is equal to unity. Only one-half of the slab is shown, the origin of the $z$-coordinate corresponding to the center of the slab and the density profiles being averaged over the two halves. The water density is roughly constant for more than 1 $\mathrm{nm}$ from the center of the slab, after which it starts to decay to 0 , marking the interfacial region. Throughout the concentration range investigated, the highly polarizable $\mathrm{SCN}^{-}$ions show surface enhancement, whereas the small nonpolarizable $\mathrm{Na}^{+}$ions are repelled from the surface. The peak interfacial concentration of $\mathrm{SCN}^{-}$is about $50 \%$ above the bulk value for the lower concentrations but decreases slightly at higher concentrations. This surface peak is accompanied by subsurface depletion and, except for the most dilute system (where the statistics are relatively poor due to the small number of ions), also by a subsurface peak of $\mathrm{Na}^{+}$.

The value of the surface enhancement of thiocyanide predicted by the present molecular dynamics simulations should be viewed as a lower bound. The reason is that we have reduced the value of the $\mathrm{SCN}^{-}$gas-phase polarizability by $22 \%$ and employed a smooth cutoff scheme for the induced dipoles. The reduction of the gas-phase polarizability can be (at least partly) 

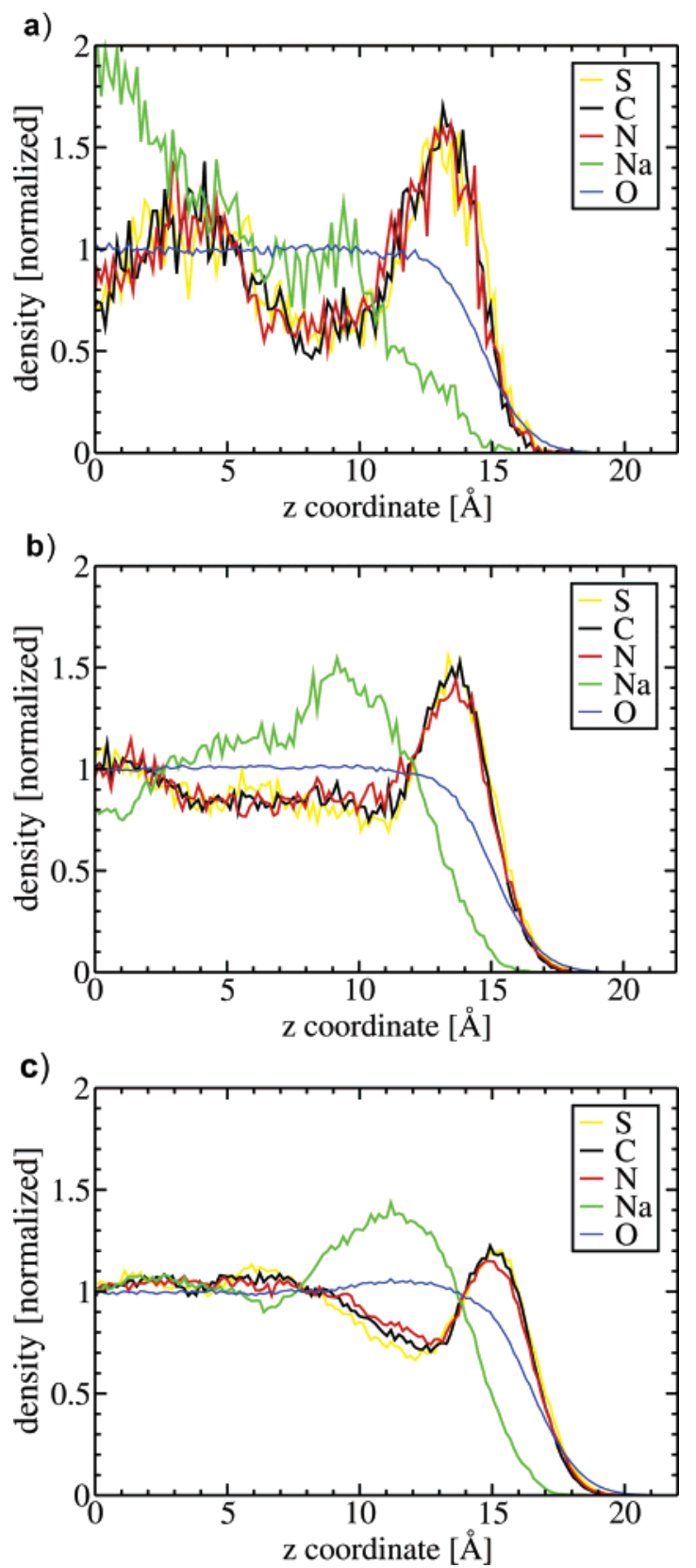

Figure 5. The density profiles of the (a) 0.6 , (b) 1.2 , and (c) $3.5 \mathrm{M}$ aqueous NaSCN solution. The blue curve is the water oxygen signal, defining the slab. The green color corresponds to the sodium cation. The yellow, black, and red curves correspond to the S, C, and N atoms of the thiocyanate anion. The densities are normalized so that the area under them is equal, with the bulk water density being one.

justified by the solvent effect but the main reason to employ the cutoff procedure was to avoid the polarization catastrophe. ${ }^{43}$ Since the propensity of ions for the air/water interface positively correlates with the ion polarizability ${ }^{25}$ our simulations tend to somewhat underestimate the surface enhancement of $\mathrm{SCN}^{-}$.

\section{Discussion}

Until recently, the description of the distribution of ions at the liquid water surface has been limited to continuum models on the theoretical side and to surface excesses (integrated surface concentrations) derived from surface tension experiments on the experimental side. ${ }^{55}$ The original continuum model by Onsager and Samaras ${ }^{13}$ described the interface as a discontinuity between two continuous dielectric media and the ions as point charges. The ions are repelled from the interface by image charge repulsion, as first suggested by Wagner. ${ }^{56}$ The continuum models have since been refined to include polarizability and ion-specific properties such as ion size and dispersion, ${ }^{14,15}$ but these still mostly predict the ions to be repelled from the outermost liquid layer at the surface. Recently, a smooth interface has been incorporated into a continuum model by relaxing the dielectric constant continuously from the bulk water value (79) to air (1) over a few angstroms. ${ }^{18}$ Interestingly, this continuum model, as well as another one that employs large values of ionic polarizability, ${ }^{19}$ predicts for highly polarizable ions a minimum in the Gibbs energy profile just below the interface, and of about the same magnitude as that following from molecular dynamics simulations. ${ }^{2,3}$ However, continuum models inherently lack a description of the asymmetry of water molecules and the directionality of hydrogen bonds, which govern the difference in the interactions of water molecules with anions and cations. Furthermore, they obviously lack the description of the granular nature of the solvent, which engenders surface roughness. These effects are naturally included in the MD simulations.

The surface tension of electrolyte solutions increases roughly linearly with concentration above $0.01 \mathrm{M}$. By the Gibbs adsorption isotherm, ${ }^{10}$ the ions are depleted from the interface accordingly. However, as a thermodynamic relation, the Gibbs equation does not take into account any microscopic structure of the ion distribution, but only the concentration integrated over the whole interfacial region. For electrolyte pairs that include a highly polarizable anion, the concentration is a strongly nonmonotonic function of the distance from the surface. The anions are enhanced, relative to the bulk concentration, at the outermost liquid layer but depleted in subsurface. In turn, small nonpolarizable cations are repelled from the topmost layer but their concentration can be enhanced in the subsurface. Nevertheless, in accord with the surface tension measurements, the total surface ion excess can be negative even though the outermost liquid layer is enhanced with the anions. ${ }^{25}$

Below $0.01 \mathrm{M}$, surface tension measurements are still controversial due to the accuracy needed to measure very small changes. Jones and Ray measured a minimum in the surface tension at $1 \mathrm{mM}$ for several salts. ${ }^{48,49}$ These measurements were later contested ${ }^{51,52}$ and remain today as a curiosity. The experimental authors of the present study have recently observed the Jones-Ray effect for iodide ${ }^{6}$ and ferrocyanide ${ }^{50}$ solutions at dilute concentrations. Straightforward molecular dynamics simulations at such low concentrations are technically hardly feasible, since there would be less than a single ion pair in a reasonably sized unit box. Nevertheless, potential of mean force calculations for a single iodide anion in a box of 1000 water molecules also indicates significant surface propensity. ${ }^{2}$

As discussed above, small nonpolarizable cations typically exhibit different surface behavior than large polarizable anions. However, recent investigations have shown that $\mathrm{H}_{3} \mathrm{O}^{+}$is an exception to the general case of cations being repelled from the surface. Recent molecular simulations showed enhanced surface affinity of a single hydronium cation to the aqueous surface, compared, e.g., to alkali cations. ${ }^{57,58}$ This prediction has recently been supported by a combined molecular dynamics and SFG investigation ${ }^{59}$ and a separate SHG study ${ }^{53}$ of concentrated acids (such as $\mathrm{HCl}, \mathrm{HBr}$, or $\mathrm{HI}$ ) which explained why the surface tension of monovalent acid solutions is lower than that of water. ${ }^{55}$ The force that brings hydronium to the interface is different from that operating for the anions. ${ }^{57,59}$ Hydronium is only capable of forming three hydrogen bonds 
since its oxygen is a poor hydrogen bond acceptor, and thus it disrupts the hydrogen bonding network in the bulk. The ion can, in this respect, be qualitatively viewed as a defect in the hydrogen bonding network, and similar to lattice defects in crystals, it is expelled to the surface where it can be accommodated more easily.

\section{Conclusions}

We have presented a combined description of thiocyanide adsorption at the liquid water-air interface by MD simulations and SHG experiments on aqueous solutions of $\mathrm{NaSCN}$ of varying concentrations. The experiments reveal a simple Langmuir behavior of the thiocyanide surface concentration with respect to the bulk. Fit of the experimental data to a Langmuir isotherm yields a Gibbs free energy of adsorption of $\mathrm{SCN}^{-}$of $-1.80 \pm 0.03 \mathrm{kcal} / \mathrm{mol}$ corresponding to an onset of surface saturation above $1 \mathrm{M}$. MD simulations in slab geometry confirm the propensity of thiocyanide for the surface of aqueous solutions. Due to the reduced polarizability and cutoff on induced dipoles in the simulations the calculated peak $50 \%$ $\mathrm{SCN}^{-}$enhancement at the surface compared to the bulk concentration should be viewed as a lower limit to the thiocianide surface affinity. The simulations also reveal a slight decrease of the surface peak of thiocyanide with increasing NaSCN concentration, confirming thus the beginning surface saturation observed in the experiment.

The description of inorganic ions in the interfacial region of the water-air interface is becoming more detailed and a consistent new picture is beginning to emerge. Small nonpolarizable (hard) ions are repelled from the aqueous surface in accord with the traditional picture based on continuum solvent models. However, polarizable (soft) anions exhibit a nonmonotonic interfacial distribution, being enhanced at the outermost surface layer, but depleted in the subsurface. At appreciable concentrations, the countercations are then attracted by the anionic surface layer, and become subsequently enhanced in the subsurface. The fact that ions can play an active role at the air-water interface and that such behavior is strongly ionspecific also has important general implications for chemical reactions occurring at surfaces of aqueous electrolytes, such as aerosol particles and ocean surfaces.

Acknowledgment. The Berkeley group is funded by the Experimental Physical Chemistry Division at the National Science Foundation. P.B.P. is funded by the Danish Research Training Council. Support to P.J. via the NSF-funded Environmental Molecular Science Institute (grants CHE 0431312 and 0209719) and from the Czech Ministry of Education (grant LC512) is gratefully acknowledged. Part of the work in Prague was supported via the Research Project Z40550506.

\section{References and Notes}

(1) Jungwirth, P.; Tobias, D. J. J. Phys. Chem. B 2001, 105, 10468

(2) Dang, L. X.; Chang, T.-M. J. Phys. Chem. B 2002, 106, 235.

(3) Jungwirth, P.; Tobias, D. J. J. Phys. Chem. B 2002, 106, 6361.

(4) Salvador, P.; Curtis, J. E.; Tobias, D. J.; Jungwirth, P. Phys. Chem. Chem. Phys. 2003, 5, 3752.

(5) Yang, X.; Kiran, B.; Wang, X.-B.; Wang, L.-S.; Mucha, M.; Jungwirth, P. J. Phys. Chem. A 2004, 108, 7820

(6) Petersen, P. B.; Johnson, J. C.; Knutsen, K. P.; Saykally, R. J. Chem. Phys. Lett. 2004, 397, 46.

(7) Petersen, P. B.; Saykally, R. J. Chem. Phys. Lett. 2004, 397, 51.

(8) Liu, D.; Ma, G.; Levering, L. M.; Allen, H. C. J. Phys. Chem. B 2004, 108, 2252.

(9) Ghosal, S.; Shbeeb, A.; Hemminger, J. C. Geophys. Res. Lett. 2000, $27,1879$.
(10) Adamson, A. W.; Gast, A. P. Physical Chemistry of Surfaces, 6th ed.; Wiley: New York, 1997.

(11) Durand-Vidal, S.; Simonin, J.-P.; Turq, P. Electrolytes at Interfaces; Kluwer Academic Publishers: Dordrecht, The Netherlands, 2000.

(12) Fawcett, W. R. Liquids, Solutions, and Interfaces; Oxford University Press: Oxford, UK, 2004.

(13) Onsager, L.; Samaras, N. N. T. J. Phys. Chem. 1934, 2, 528

(14) Karraker, K. A.; Radke, C. J. Adv. Colloid Interface Sci. 2002, 96 , 231.

(15) Manciu, M.; Ruckenstein, E. Adv. Colloid Interface Sci. 2003, 105, 63.

(16) Markin, V. S.; Volkov, A. G. J. Phys. Chem. B 2002, 106, 11810.

(17) Bostrom, M.; Williams, D. R. M.; Ninham, B. W. Langmuir 2001, 17,4475 .

(18) Frediani, L.; Mennucci, B.; Cammi, R. J. Phys. Chem. B 2004, $108,13796$.

(19) Levin, Y. Submitted for publication

(20) Kunz, W.; Lo Nostro, P.; Ninham, B. W. Curr. Opin. Colloid Interface Sci. 2004, 9, 1 .

(21) Hu, J. H.; Shi, Q.; Davidovits, P.; Worsnop, D. R.; Zahniser, M. S.; Kolb, C. E. J. Phys. Chem. 1995, 99, 8768.

(22) Knipping, E. M.; Lakin, M. J.; Foster, K. L.; Jungwirth, P.; Tobias, D. J.; Gerber, R. B.; Dabdub, D.; Finlayson-Pitts, B. J. Science 2000, 288 , 301.

(23) Laskin, A.; Gaspar, D. J.; Wang, W.; Hunt, S. W.; Cowin, J. P.; Colson, S. D.; Finlayson-Pitts, B. J. Science 2003, 301, 340.

(24) Raymond, E. A.; Richmond, G. L. J. Phys. Chem. B 2004, 108, 5051.

(25) Vrbka, L.; Mucha, M.; Minofar, B.; Jungwirth, P.; Brown, E. C. Tobias, D. J. Curr. Opn. Colloid Interface Sci. 2004, 9, 67.

(26) Petersen, P. B.; Saykally, R. J. In preparation.

(27) Shen, Y. R. The Principles of Nonlinear Optics; Wiley: New York, 1984

(28) Boyd, R. W. Nonlinear Optics, 2nd ed.; Elsevier: Amsterdam, The Netherlands, 2003.

(29) Eisenthal, K. B. Chem. Rev. 1996, 96, 1343.

(30) Richmond, G. L. Annu. Rev. Phys. Chem. 2001, 52, 357.

(31) Sokhan, V. P.; Tildesley, D. J. Mol. Phys. 1997, 92, 625.

(32) Simpson, G. J.; Rowlen, K. L. Anal. Chem. 2000, 72, 3407.

(33) Dukhin, S. S.; Kretzschmar, G.; Miller, R. Dynamics of Adsorption at Liquid Interfaces. Theory, Experiment, Application; Elsevier: Amsterdam, The Netherlands, 1995.

(34) Chang, C.-H.; Franses, E. I. Colloids Surf. A 1995, 100, 1.

(35) Prosser, A. J.; Franses, E. I. Colloids Surf. A 2001, 178, 1.

(36) Wilson, M. A.; Pohorille, A. J. Chem. Phys. 1991, 95, 6005.

(37) Caldwell, J. W.; Kollman, A. J. Phys. Chem. 1995, 99, 6208

(38) Perera, L.; Berkowitz, M. L. J. Chem. Phys 1991, 95, 1954.

(39) Breneman, C. M.; Wiberg, K. B. J. Comput. Chem. 1990, 11, 361

(40) Sansone, R.; Ebner, C.; Probst, M. J. Mol. Liq. 2000, 88, 129.

(41) Darden, T.; York, D.; Pedersen, L. J. Chem. Phys. 1993, 98, 10089.

(42) Jungwirth, P.; Tobias, D. J. J. Phys. Chem. A 2002, 106, 379.

(43) Thole, B. T. Chem. Phys. 1981, 59, 341.

(44) Berendsen, H. J. C.; Postma, J. P. M.; DiNola, A.; Haak, J. R. J. Chem. Phys. 1984, 81, 3684.

(45) Ryckaert, J. P.; Cicotti, G.; Berendsen, H. J. C. J. Comput. Phys. 1977, 23, 327

(46) Case, D. A.; Darden, T. A.; Cheatham, T. E., III; Simmerling, C L.; Wang, J.; Duke, R. E.; Luo, R.; Merz, K. M.; Wang, B.; Pearlman, D. A.; Crowley, M.; Brozell, S.; Tsui, V.; Gohlke, H.; Mongan, J.; Hornak, V.; Cui, G.; Beroza, P.; Schafmeister, C.; Caldwell, J. W.; Ross, W. S.; Kollman, P. A. AMBER 8; University of California: San Francisco, CA 2004.

(47) Frisch, M. J.; Trucks, G. W.; Schlegel, H. B.; Scuseria, G. E.; Robb, M. A.; Cheeseman, J. R.; Montgomery, J. A., Jr.; Vreven, T.; Kudin, K. N.; Burant, J. C.; Millam, J. M.; Iyengar, S. S.; Tomasi, J.; Barone, V.; Mennucci, B.; Cossi, M.; Scalmani, G.; Rega, N.; Petersson, G. A. Nakatsuji, H.; Hada, M.; Ehara, M.; Toyota, K.; Fukuda, R.; Hasegawa, J.; Ishida, M.; Nakajima, T.; Honda, Y.; Kitao, O.; Nakai, H.; Klene, M.; Li, X.; Knox, J. E.; Hratchian, H. P.; Cross, J. B.; Bakken, V.; Adamo, C.; Jaramillo, J.; Gomperts, R.; Stratmann, R. E.; Yazyev, O.; Austin, A. J.; Cammi, R.; Pomelli, C.; Ochterski, J. W.; Ayala, P. Y.; Morokuma, K.; Voth, G. A.; Salvador, P.; Dannenberg, J. J.; Zakrzewski, V. G.; Dapprich, S.; Daniels, A. D.; Strain, M. C.; Farkas, O.; Malick, D. K.; Rabuck, A. D.; Raghavachari, K.; Foresman, J. B.; Ortiz, J. V.; Cui, Q.; Baboul, A. G.; Clifford, S.; Cioslowski, J.; Stefanov, B. B.; Liu, G.; Liashenko, A.; Piskorz, P.; Komaromi, I.; Martin, R. L.; Fox, D. J.; Keith, T.; Al-Laham, M. A.; Peng, C. Y.; Nanayakkara, A.; Challacombe, M.; Gill, P. M. W.; Johnson, B.; Chen, W.; Wong, M. W.; Gonzalez, C.; Pople, J. A. Gaussian 03; Gaussian, Inc.: Wallingford, CT, 2004.

(48) Jones, G.; Ray, W. A. J. Am. Chem. Soc. 1937, 59, 187.

(49) Jones, G.; Ray, W. A. J. Am. Chem. Soc. 1941, 63, 288.

(50) Petersen, P. B.; Saykally, R. J. In preparation.

(51) Langmuir, I. Science 1938, 88, 430. 
(52) Langmuir, I. J. Chem. Phys. 1938, 6, 873.

(53) Petersen, P. B.; Saykally, R. J. J. Phys. Chem. B 2005, 109, 79767980.

(54) Wang, H.; Borguet, E.; Eisenthal, K. B. J. Phys. Chem. B 1998, $102,4927$.

(55) Weissenborn, P. K.; Pugh, R. J. J. Colloid Interface Sci. 1996, 184, 550 .
(56) Wagner, V. C. Phys. Z. 1924, 15, 474.

(57) Petersen, M. K.; Iyengar, S. S.; Day, T. J. F.; Voth, G. A. J. Phys. Chem. B 2004, 108, 14804.

(58) Dang, L. X. J. Chem. Phys. 2003, 119, 6351.

(59) Mucha, M.; Frigato, T.; Levering, L.; Allen, H. C.; Tobias, D. J.; Dang, L. X.; Jungwirth, P. J. Phys. Chem. B 2005, 109, 7617-7623. 